\title{
Defects and their range in pure bismuth irradiated with swift Xe ions studied by positron annihilation techniques
}

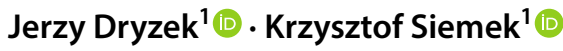

Received: 6 November 2018 / Accepted: 24 December 2018 / Published online: 8 January 2019

(c) The Author(s) 2019

\begin{abstract}
Investigations of defects and their spatial distribution in $\mathrm{Bi}$ irradiated with $167 \mathrm{MeV} \mathrm{Xe}{ }^{26+}$ ions of different doses have been performed using conventional positron lifetime spectroscopy and variable energy positron beam. In an implanted layer, in which ions are traveling, interacting with atoms and stopping, only clusters which consist of more than eight vacancies were found. It was assigned from ab initio theoretical calculations of positron lifetime in vacancy clusters in Bi. The thickness of this layer corresponds to the range of implanted ions calculated from the SRIM code. However, beyond this layer, an extended layer with such defects has also been found. Its thickness is comparable to the thickness of the implanted layer and it depends on the dose. Defects induced by implantation are also present near the entrance surface, and their concentration depends on the dose of implanted ions as well. Three methods for reconstructing the actual mean positron lifetime and thus the induced depth defect distribution have been proposed, two of them are used in current research.
\end{abstract}

\section{Introduction and motivation}

Many aspects of swift ion-solid interactions have been intensively studied, including the morphology of atomic defects generated during this interaction [1]. This is important because they play a key role in the constitution of the final physical properties of the region exposed to this interaction. It is generally accepted that defects are generated mainly at the end of the ion track results from inelastic nuclear collision cascades when ion energy is of the order of $\mathrm{keV}$. This takes place in the nuclear stopping power regime. However, they can also occur in the electronic regime, i.e., during ionization and excitation of electrons, when ion energy is much higher. According to the thermal spike model, strong coupling of excited electrons with phonons results in rapid heating and cooling of the material in the vicinity of the track. This leads to a transient and highly disordered zone [2]. Damage of the target takes place also at the entrance surface when the target atoms are spattered [3]. Thus, implantation of swift ions into target results in a generation of damages ranging from point defects to phase transition. It is accepted, that the thickness of the damaged

Jerzy Dryzek

jerzy.dryzek@ifj.edu.pl

1 Institute of Nuclear Physics Polish Academy of Sciences, 31342 Kraków, Poland layer is from nanometers to a dozen micrometers, which overlaps with the ion projectile range $\left(R_{\mathrm{d}}\right)$.

The magnitude of the damage induced depends not only on the irradiation conditions, i.e., the incident energy of ions, their charge and the fluence but also on the physical and chemical properties of the irradiated target material. The response to irradiation must be different depending on the material, even under the same conditions. Here the question arises about the depth distribution of the generated defects. The theoretical simulations based, for instance, on the SRIM/TRIM code indicate that along the track the vacancies concentration is almost constant but near the end, the track gradually increases and drops to zero at or beyond the ion projectile range [4]. The theoretical distribution has a characteristic peak at the end of the track, in the region of the Bragg peak of the curve for electronic stopping power. Nevertheless, experiments have shown a much complex shape of the distribution which additionally is extended much beyond the projectile range [5]. This was detected by experimental techniques such as TEM or microhardness and also positron annihilation techniques. Sharkeev et al. even proposed to call this as the long-range effect (LRE) [6]. However, there is no consensus about the existence of this. Nevertheless, we argue that the positron annihilation technique, extremely sensitive to the defects can be a proper tool to confirm or reject this effect. 
Positron annihilation (PA) spectroscopy has been used to study implantation-induced defects mainly in semiconductors [7], however, also in other materials (see for instance Ref. [8]). Positrons are uniquely sensitive to the open volume defects like vacancies and their clusters, voids or dislocations. Additionally, by means of variable energy positron beam technique it is also possible to obtain the distribution or profile of these defects below the surface [9]. However, this technique allows testing to a depth of only one or two micrometers. Recently, using the conventional positron source, i.e., ${ }^{22} \mathrm{Na}$ we applied the experimental technique which allows us to perform tests at the depth of dose micrometers [10]. This is suitable for profiling defects induced by irradiation with $\mathrm{MeV}$ ions as it was shown in our recent studies. We have carried out several such studies so far where metals like $\mathrm{Cu}$ [11], $\mathrm{Ag}$ [12], $\mathrm{Fe}$ [13] and $\mathrm{Ti}$ [14] were irradiated with swift $\mathrm{Xe}^{26+}$ ions of energy about $167 \mathrm{MeV}$. In the case of $\mathrm{Ag}$ and $\mathrm{Fe}$, defects were observed far beyond the projectile range, but in the case of $\mathrm{Cu}$ and $\mathrm{Ti}$, the depth of the damage layer correlated exactly with the range of ions. We did not confirm the total range of damage extended about three orders than the projectile range reported by some authors $[6,15,16]$, however, we have not ruled out the possibility of such a large depth of damage. It can be assumed that samples' preparation and irradiation procedure can generate the damage at a larger distance than expected, i.e., $R_{\mathrm{d}}$. The finite element analysis calculations have pointed out that high stress, significantly above the yield strength that can occur during implantation, may extend plastic deformation beyond the projectile range [13]. In this paper, we intend to report similar studies of defect distribution not in metal but in semimetal, i.e., polycrystalline $\mathrm{Bi}$ irradiated with swift $\mathrm{Xe}^{26+}$ ions.

Many authors have studied Bi bulk samples and thin films irradiated with different ions. Dufour et al. irradiated with swift Xe and Ta ions Bi samples at several temperatures between 20 and $300 \mathrm{~K}$ and they monitored electrical resistivity as the function of fluence [3]. They have shown that the damage efficiency and ions track radii increase as the irradiation temperature increases. This supports the thermal spike model, i.e., the initial kinetic energy transfers to target atoms mainly by the electronic excitation.

It should also be reminded of other studies involving irradiated Bi. Its foils placed in lead target irradiated by $660 \mathrm{MeV}$ protons were applied for production of Po isotopes. The maximum of production of these isotopes was observed on the end of a range of protons when their energy is less than $100 \mathrm{MeV}$ [17]. Many authors studied the spattering yields from $\mathrm{Bi}$ thin films bombarded by swift $\mathrm{Cu}^{4+}$ and $\mathrm{Cu}^{7+}$ ions [18] and $\mathrm{Ar}^{+}$ions [19]. They found a satisfactory agreement with Monte Carlo simulation using the SRIM 2008 code.
In this article, we present positron annihilation research in polycrystalline, well-annealed samples of $\mathrm{Bi}$ exposed to irradiation by $\mathrm{Xe}^{26+}$ ions with $167 \mathrm{MeV}$ of different fluencies. Conventional positron lifetime measurement will be used to determine the depth profile of defects induced by these ions irradiation. The two proposed methods allow us to reconstruct the actual defect depth profile. The region close to the surface of the irradiated samples will be characterized by the slow positron beam technique. To recognize the type of defect, the ab initio calculations of positron lifetime in vacancy clusters are presented. In general, the purpose of our research is to confirm previous arrangements for LRE, but in semimetal Bi.

\section{Experimental details}

\subsection{Sample preparation}

Samples of pure Bi (99.997\% purity purchased from Goodfellow) had the shape of a plate with a height of $2 \mathrm{~mm}$ and a diameter of $10 \mathrm{~mm}$ and prior to irradiation, they were annealed in an $\mathrm{N}_{2}$ gas flow at $200{ }^{\circ} \mathrm{C}$ for $1 \mathrm{~h}$ and then slowly cooled to room temperature. To clean the surface, all samples were etched in a $25 \%$ solution of nitric acid in distilled water and their thickness was reduced by $50 \mu \mathrm{m}$. The measurements of the positron lifetime spectrum for such virgin samples revealed only one component equal to $241 \pm 1$ ps which corresponds well with the experimental bulk value reported, i.e., 241 ps, [20], 249 ps [21], 240 ps [22]. This proves that only residual defects remain in the samples, which are not actually visible to positrons.

The implantation process of the samples was performed at IC-100 cyclotron at Flerov Laboratory of Nuclear Reactions at Joint Institute for Nuclear Research (JINR) in Dubna, Russia. $\mathrm{Xe}^{26+}$ heavy ions with an energy of $167 \mathrm{MeV}$ and three doses, namely $10^{13}, 5 \times 10^{13}$ and $10^{14}$ ions $/ \mathrm{cm}^{2}$ were applied. The average ion flux was $5 \times 10^{9} \mathrm{~cm}^{-2} \mathrm{~s}^{-1}$. The temperature of the samples in their volume during irradiation did not exceed $80{ }^{\circ} \mathrm{C}$.

\subsection{Positron measurements}

The present work used conventional fast-fast coincidence digital positron lifetime spectrometer APV8702 (made by TechnoAP Co. Ltd.). As the detector, two photomultipliers H3378-50 (made by Hamamatsu) coupled with $\mathrm{BaF}_{2}$ scintillators were applied. The time resolution of the spectrometer (FWHM) was about 180 ps. The isotope ${ }^{22} \mathrm{Na}$ was used as the positron source encapsulated into a titanium foil $7 \mu \mathrm{m}$ thick, with an activity of about $27 \mu \mathrm{Ci}$. Two identical prepared samples were placed on both sides of the positron source. 
With an approximation, the depth profile of the implanted positrons, i.e., the depth distribution where positrons stop, as they are already thermally and practically annihilated, is expressed by the exponential decay function characterized by only one parameter, linear absorption coefficient. For $\mathrm{Bi}$, its value is about $435 \mathrm{~cm}^{-1}$, so about $63 \%$ of positrons emitted from this source are stopped in a layer with a thickness of $23 \mu \mathrm{m}$ [23]. At closer inspection, closer to the surface, at a depth of less than $10 \mu \mathrm{m}$, the profile changes shape, and becomes steeper (see e.g. Ref. [24]). The linear absorption coefficient value in this region increases about factor of two. So, this convinces us that using conventional positron techniques with ${ }^{22} \mathrm{Na}$ positrons, one can study the near-surface regions, at the depth of about $10 \mu \mathrm{m}$. Indeed, such studies are possible and this was shown in our previous papers devoted to samples exposed to implantation with swift $\mathrm{Xe}^{26+}$ ions [13].

Slow positron beam equipment called as the variable energy positron beam (VEP), suitable for studies of the damages induced by irradiation at a depth less than $1 \mu \mathrm{m}$, was used at JINR in Dubna. The energy of injected positrons was between 0.1 and $35 \mathrm{keV}$. The beam intensity was about $10^{5} \mathrm{e}^{+} / \mathrm{s}$ and its diameter was $5 \mathrm{~mm}$. The HPGe detector with an energy resolution of $1.20 \mathrm{keV}$ at $511 \mathrm{keV}$ was used to monitor an annihilation line shape parameter, S-parameter, in relation to positron incident energy. This parameter is defined as the ratio of the area below the central part of the annihilation peak to the total area in the compartment of this line. The energy interval taken for calculation is always constant within the whole measurement session.

\section{Experimental results}

\subsection{Positron lifetime depth profiles}

To detect the depth profile of defects resulting from implantation, the sample was etched to remove a layer of about $2 \mu \mathrm{m}$ thick and then the positron lifetime spectrum was measured. $25 \%$ solution of nitride acid in distilled water was chosen as an etching solution. The thickness of the sample was measured before and after etching using a digital micro-screw with an accuracy of $\pm 0.5 \mu \mathrm{m}$. The procedure was subsequently repeated until the bulk value of the positron lifetime was obtained, this means that the damage layer has been removed and the virgin region has been reached. In practice, this procedure allows us to scan the sample to any depth, although it should be mentioned that the sample will be destroyed after such measurement. Initially, near the entrance surface, in each spectrum, two lifetime components $\tau_{1}$ and $\tau_{2}$ were resolved. Their values and intensities, $I_{1}$ and
$I_{2}$ (in percentage) will be analyzed. However, it is convenient to define the mean positron lifetime value as follows:

$\bar{\tau}(z)=\left(\tau_{1} I_{1}+\tau_{2} I_{2}\right) / 100 \%$.

Deeper only single lifetime component was resolved from the measured spectra, and this value will be treated as the mean positron lifetime too. The mean positron lifetime is sensitive to the presence of the open volume defects. When these defects occur in a test sample, its value increases significantly above the bulk value. It is a solid parameter, which is an indicator of the presence of open volume defects [7].

The projectile range, $R_{\mathrm{d}}$, of implanted $\mathrm{Xe}^{26+}$ ions into Bi was obtained using SRIM code [4], its value was about $13.2 \mu \mathrm{m}$ and longitudinal straggle about $1 \mu \mathrm{m}$. In Fig. 1a, the shadow rectangular represents the implanted layer (IL), i.e., a region where ions travel and interact with target atoms. However, in this figure, we depicted the theoretical depth distribution of vacancies generated by recoil obtained by this code. $10^{6}$ ions were used in the SRIM code simulations, but the obtained results were recalculated to obtain the corresponding dose used in the implantation. It is clearly visible that the number of vacancies increases from the entrance surface and is maximal at the end of the ion tracks. The maximum height is proportional to the dose, as expected.

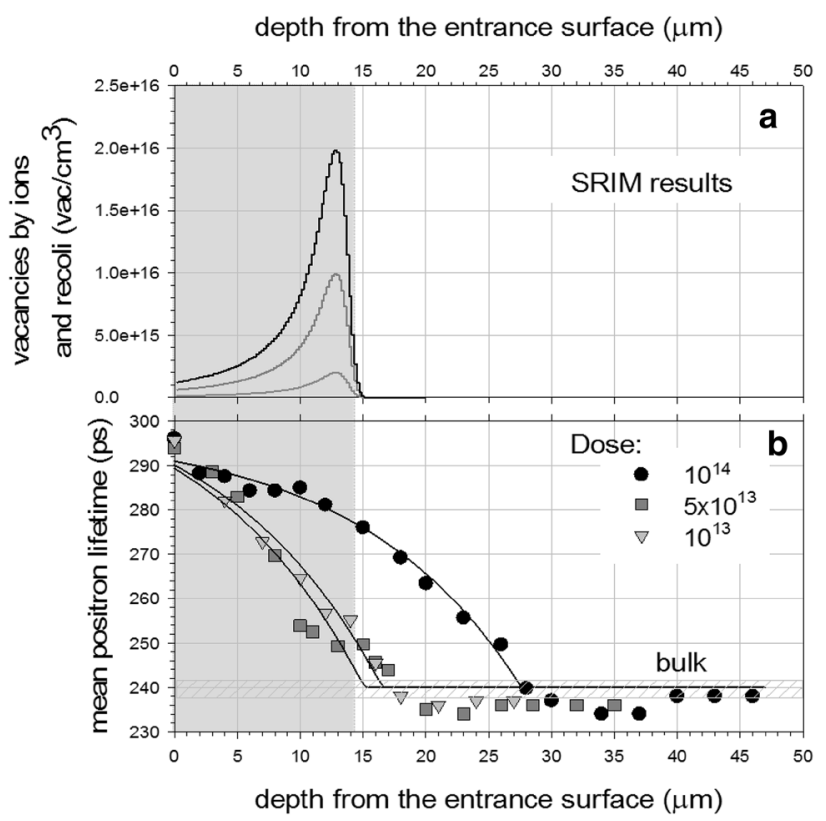

Fig. 1 SRIM simulation results (a) of vacancies' density in dependency on depth from the entrance surface after $167 \mathrm{MeV} \mathrm{Xe} \mathrm{X}^{26+}$ implantation with different doses in Bi. The depth distribution of the measured mean positron lifetimes (b) obtained in the etching experiment as the function of depth from the entrance surface. The hatched region represents the value of positron lifetime resolved for a wellannealed specimen. The gray rectangular represents the region of the implanted layer, where ions are traveling and finally stop. The solid lines represent the best fit of Eq. (3) to the experimental points for each dose 
This is clear, because only ions are the source of damages, if more ions are implanted, then more damages, and defects are present. In Fig. 1b, the measured depth profile of the mean positron lifetime is shown for all three doses. In the region of the IL, the mean positron lifetime decreases gradually. However, the bulk value of the mean positron lifetime, tagged by hatched rectangular is obtained above $R_{\mathrm{d}}$. For the highest dose, this was at the depth of about $28 \mu \mathrm{m}$, i.e., about two times more than $R_{\mathrm{d}}$. This clearly indicates that the damage layer involved by ions is extended much beyond the IL. For the lower doses, this effect is also well visible, however, the depth at which the bulk value is obtained is slightly lower, i.e., $18 \mu \mathrm{m}$, for both cases.

The obtained profiles, Fig. 1b, are the result of convolution of the positron implantation profile with the actual profile of the mean positron lifetime, which in turn is associated with the profiles of open volume defect concentration caused by implantation. This is clearly represented by Eq. (4) in Appendix 1 where a detailed discussion is given too. The approximated reconstruction of the actual profile can be carried out using Eq. (7), and the results are depicted in Fig. 2. This profile presents the actual value of the mean positron lifetime in a layer with a thickness of approximately $5 \mu \mathrm{m}$. The fraction of positrons $w$ in Eq. (7) which annihilate in the adjacent to the positron source layer is about $23 \%$, this value was calculated using the LYS-1 code $[25,26]$, where the fraction of positrons emitted from ${ }^{22} \mathrm{Na}$ in a stack of layers with different thicknesses is evaluated. The general features of the profiles of Fig. 1b are also reproduced in Fig. 2, i.e., cut off at the certain depth that exceeds the projectile range and the small reduction of the mean positron lifetime with increasing depth. However, the obtained positron lifetime values are about $30-80$ ps larger than those of Fig. 1b. This is understandable, as discussed in Appendix 1 and illustrated in the typical examples of profiles in Fig. 5, Appendix 1. It should be noted that such a reconstruction is subject to great uncertainty mostly caused by uncertainty in thickness measurements ca. $0.5 \mu \mathrm{m}$, because it could influence the determination of the $w$ value which could differ around $\pm 10 \%$. Additionally, the reconstructed value depends on the inverse of the $w$ value, which is much smaller than the unity, Eq. (7).

It seems that another more accurate approach may be proposed, in which the experimental relationships of Fig. $1 \mathrm{~b}$ can be described directly by means of Eq. (4). However, in this case, one should know what kind of dependency should be expected for the actual mean positron lifetime. Looking at examples of dependencies of Fig. 5 in Appendix 1, it seems that the dependency in Fig. 5a (solid line) best suits the experimental dependencies. Let us assume then that the actual dependency of the mean positron lifetime is described as a step-like function as follows:

$\bar{\tau}(z)=a[1-\theta(z-b)]+c$,

where $\theta(z)$ is the Heaviside step function, and $a, b$ and $c$ are the adjustable parameters. From Eq. (4), the following relation can be obtained for the mean positron lifetime convoluted with the positron implantation profile represented by Eq. (5)

$\bar{\tau}_{\mathrm{m}}(z)=a\left\{\theta(z-b)\left[\mathrm{e}^{\mu(z-b)}-1\right]-\mathrm{e}^{\mu(z-b)}\right\}+a+c$.

The solid lines in Fig. 1b represent the best fits of this relation to the experimental points for each dose. The width of the step, $b$ parameter is equal to $15.1 \pm 0.7,16.5 \pm 0.5$ and $27.6 \pm 0.6 \mu \mathrm{m}$, for dose $10^{13}, 5 \times 10^{13}$ and $10^{14}$ ions/
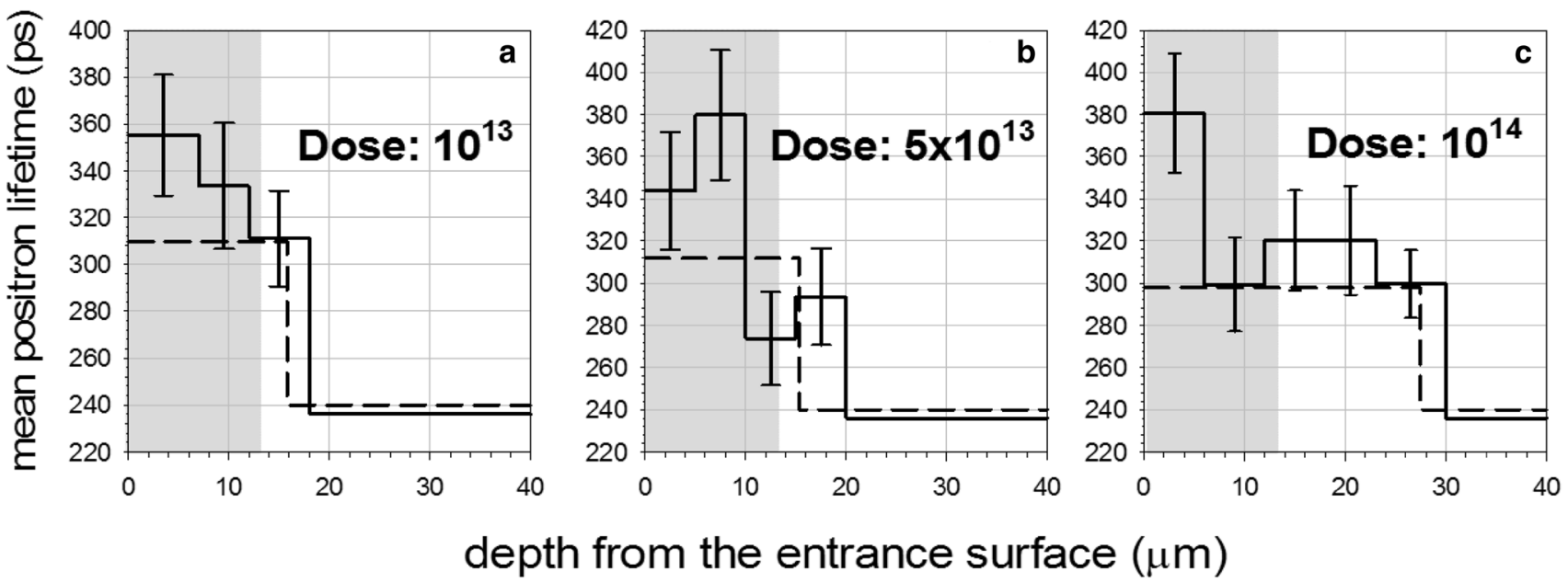

Fig. 2 The reconstructed value, according to Eq. (7), and the depth dependency (solid line) of mean positron lifetime in the layer of thickness of about $5 \mu \mathrm{m}$, for Bi samples implanted with $\mathrm{Xe}^{26+}$ ions of $167 \mathrm{MeV}$ with different doses. For this, the depth dependencies from

Fig. 1 were applied. The gray rectangular represents the region of the implanted layer, where ions are traveling and finally stop. The dashed lines represent the step function, Eq. (2) results from fit of Eq. (3) to the experimental points from Fig. $1 b$ 
$\mathrm{cm}^{2}$, respectively. The parameter $a$ representing step height is equal to $69.6 \pm 3,71.8 \pm 2,57.9 \pm 1.6$ ps for dose $10^{13}$, $5 \times 10^{13}$ and $10^{14}$ ions $/ \mathrm{cm}^{2}$, respectively. The value of the $c$ parameter was fixed and equal to $240 \mathrm{ps}$. The linear absorption coefficient $\mu$ is also an adjustable parameter, its value obtained from the fits is about $565 \pm 23 \mathrm{~cm}^{-1}, 730 \pm 91 \mathrm{~cm}^{-1}$ and $806 \pm 80 \mathrm{~cm}^{-1}$, for dose $10^{14}, 5 \times 10^{13}$ and $10^{13}$ ions/ $\mathrm{cm}^{2}$, respectively. However, in the dependencies presented in Fig. 1b, its value was fixed at average value, i.e., equal to $700 \mathrm{~cm}^{-1}$, and it does not change $\chi^{2}$ in the fitting procedure which was above 0.95 . The fact that the above values are higher than $435 \mathrm{~cm}^{-1}$, referred to above, confirmed difference of absorption coefficient observed, see Refs. [23, 24]. Thus, this approach indicates that the actual mean positron lifetime, and hence defects can be distributed uniformly at the depth beyond $R_{\mathrm{d}}$. Generally, this is not contradicted to the results obtained from reconstruction, in Fig. 2, the dashed lines represent the step dependency Eq. (2) obtained from the fits. Certainly, Eq. (2) can only be considered as an approximation of the actual dependence, another function may also be used, however, without a clear physical interpretation of adjustable parameters. Nevertheless, the most important conclusion obtained in this approach is that with increasing dose the sum thickness of IL and ELD increases, i.e., the $b$ parameter, while the step height, the $a$ parameter does not change. A slight effect was also observed in $\mathrm{Ag}$, to which $\mathrm{Xe}^{26+}$ ions are also irradiated, see Fig. 3 in Refs. $[12,13]$. But, it was not the same as a result of the Bi case in this work. It should be mentioned that this effect was also observed by Lu et al. [5] in nickel, $\mathrm{NiCo}$ and $\mathrm{NiFe}$ after irradiation of $3 \mathrm{MeV}$-charged $\mathrm{Au}$ ions. The total range of defects induced by the irradiation was equal to ca. $450 \mathrm{~nm}$ for doses $2 \times 10^{13}$ ions $/ \mathrm{cm}^{2}$, and $1100 \mathrm{~nm}$ for $5 \times 10^{15}$ ions/ $\mathrm{cm}^{2}$ as in Ni single crystal. Therefore, not only the energy of the implanted ions, as shown in Ref. [13], but also the dose has a large impact on the distribution of defects beyond the IL.

The presence of defects in the IL is obvious, but their presence outside it is a surprising result. In the literature, the authors suggest several explanations for this phenomenon. According to $\mathrm{Lu}$ et al., the extended layer of damage (ELD) can result from the diffusion of point defects from the IL into deeper regions [5]. However, our previous research and also other authors did not confirm this. Annealing an implanted iron sample resulted in the disappearance of ELD instead of its expansion [13], but, as presented by other authors, implantation at low temperature also induces ELD [27]. Sharkeev and Kozlova pointed out that mechanical stress caused by ion implantation may induce the generation of defects beyond IL [16]. Two reasons justify this explanation. The model using the finite element analysis method explains qualitatively the expansion of defects outside IL observed by

depth from the entrance surface $(\mu \mathrm{m})$
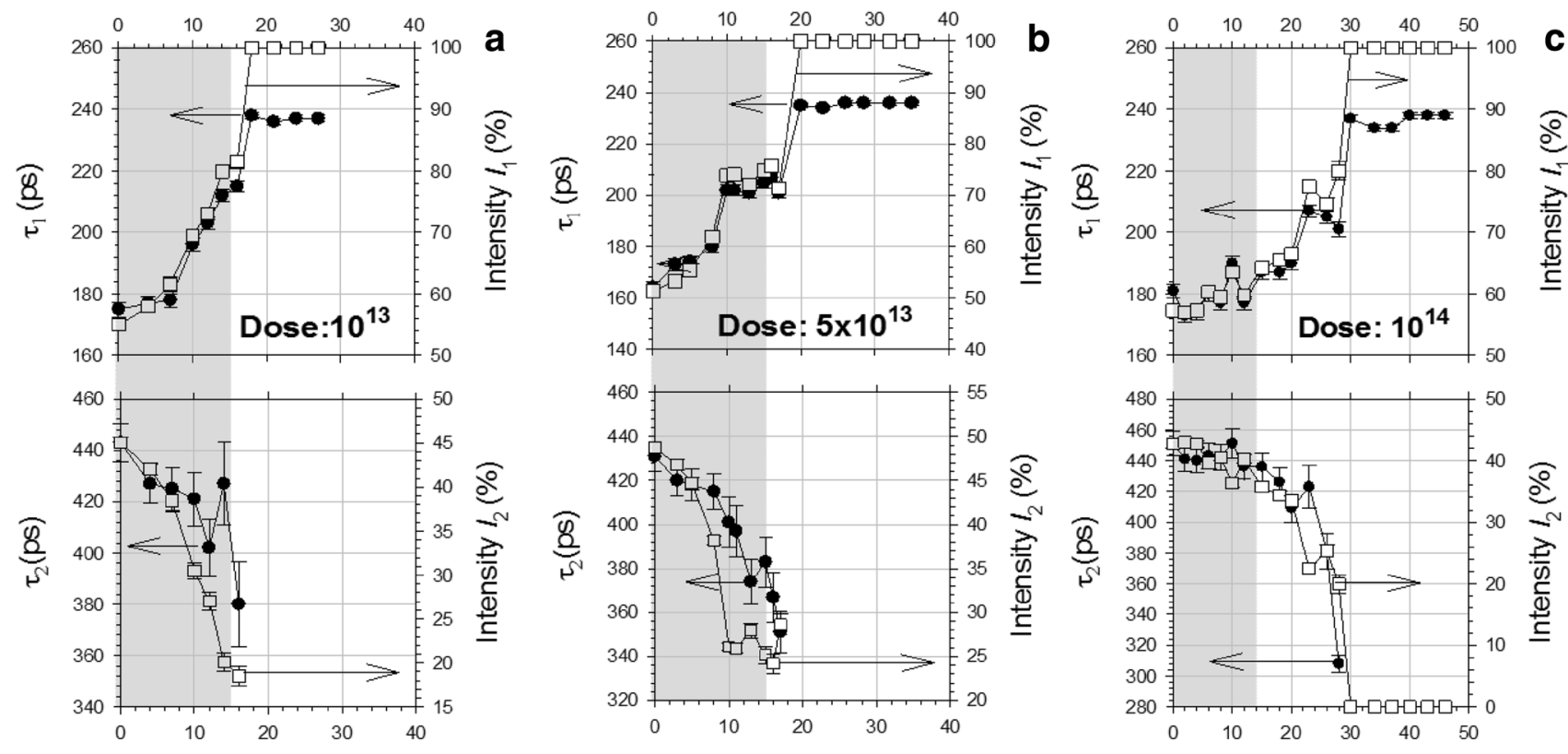

depth from the entrance surface $(\mu \mathrm{m})$

Fig. 3 The values of the positron lifetime components, $\tau_{1}, \tau_{2}$ (closed circles) and their intensities (open squares) resolved from the measured positron lifetime spectra as the function of the depth from the entrance surface of $\mathrm{Bi}$ samples irradiated with $\mathrm{Xe}^{26+}$ ions with doses: $10^{13}(\mathbf{a}), 5 \times 10^{13}(\mathbf{b})$ and $10^{14}(\mathbf{c})$ ions $/ \mathrm{cm}^{2}$. The values were used to calculate the mean positron lifetime according to Eq. (1) and presented in Fig. 1b. The gray rectangle represents the implanted layer, whose thickness was calculated from the SRIM code 
positrons [13]. The implantation process causes tensile stress in IL, but deeper layers where ions do not reach anymore are consistently squeezed. If the yield strength is exceeded in both layers, then plastic deformation will cause defects in both IL and ELD. It is important to note that they may overlap defects generated in the nuclear collision cascade. This may explain why the shape of the defect distribution as seen by positron in Figs. 1b and 2, does not correspond to the distribution obtained by the SRIM code, Fig. 1a. In this case, the concentration of defects increases with increasing depth, which should be reflected in the increase in the mean positron lifetime, which can be seen in the example in Fig. $5 \mathrm{~b}$ or $\mathrm{d}$. It is also puzzling that close to the surface of the actual mean positron lifetime and thus the concentration of defects, is greater than deeper, Fig. 2 (solid lines). Near the surface, the energy of penetrating ions is high and stopping power by nuclear collision is low, thus one should expect a low amount of defects and hence a lower value of the mean positron lifetime. The presence of tensile stress across the IL can explain this. It can be generated by increasing the temperature during implantation and lattice expansion. This can happen when the sample, e.g., is not sufficiently cooled. The presence of slowed ions can lead to swelling of the IL, and this can be another source of tensile stresses. In the finite element analysis, we assumed the tensile stress has a constant value, in fact, the depth distribution is not excluded [13]. Thus, the results of Fig. 1b, and Fig. 2 may indicate that another defect generation mechanism works in all its IL volume.

To identify the type of defects generated by implantation in $\mathrm{Bi}$ in Fig. 2, we present the values of individual positron lifetime components and their intensity obtained for all the irradiated samples. It should be noted that the value of $\tau_{1}$, closed circles in Fig. 3, is smaller than bulk value, i.e., 241 ps to a depth of approximately $30 \mu \mathrm{m}$. For a smaller depth, this value decreases to about $180 \mathrm{ps}$ on the entrance surface. The fact that the $\tau_{1}$ value is smaller than the bulk value, is well-explained by the two-state trapping model (see for instance Ref. [7]). According to this model, positrons can annihilate, both in a free and bound state, i.e., trapped in a defect. In other words, there is a bulk region in a sample in which positrons move freely and defects that trap them. The $\tau_{1}$ value is the reciprocal of the annihilation rate, the latter being the sum of the annihilation rate in the free state, i.e., in bulk and the trapping rate on the defect. Because trapping rate is proportional to the defect concentration, then with its increase $\tau_{1}$ value decreases. We can conclude that the increase of the $\tau_{1}$ value, shown in Fig. 3 indicates a reduction in defect concentration with increasing depth. This reduction is smaller in the IL than in EDL, as it is well visible for the sample irradiated with the highest dose, Fig. 3 (right). The intensity of this lifetime component, open squares in Fig. 3, also increases with increasing depth, also indicates this trend. Two-state trapping model assumes that only one type of defect is present in the sample, the value of the second lifetime $\tau_{2}$, closed circles in Fig. 3 is associated with this defect. In the IL, this value is about 440 ps for the sample with the highest dose, however, for the samples with lower doses, its value is slightly smaller about 420 ps. Such a large value suggests that cluster which consists more than eight vacancies are present in the IL (see Appendix 2, Fig. 6). It is possible to indicate a high intensity of the second lifetime component of about $45 \%$, at the entrance surface for all samples, open squares in Fig. 3. Its value decreases rapidly in the IL. In the case of the sample irradiated with the highest dose, also in the EDL, this component is observed. Thus, irradiation with fast $\mathrm{Xe}^{26+}$ ions induces the formation of large vacancy clusters, whose concentration decreases with increasing depth from the entrance surface.

The intensity of the second lifetime component can be compared with the results of the positron lifetime measurement on the worn surface of Bi sample exposed to dry sliding in the friction process [28]. In this case, the intensity was much lower, around $20 \%$, but the value positron lifetime value was almost the same, i.e., about 420 ps. Also, in this case, the value of the first lifetime component was lower than bulk value, i.e., about 230 ps. Certainly, the process of implantation and friction is completely different, but Bi's structural response leads to similar defects. As is known, in the case of friction, the surface and region below are exposed to a stress. This may suggest that static and dynamic stresses are present during implantation, but much larger, as it was reported by several authors $[16,29,30]$. It is difficult to estimate its value from positron results, because only for very small values one can expect a linear relationship between the tensile stress and the mean positron lifetime. At high stress, this relationship becomes saturated. In our last investigation, we suggested that the stress in the IL can be an order of about $1 \mathrm{GPa}$, however, in Bi this value can be different.

\subsection{VEP results}

The results obtained using conventional positron lifetime spectroscopy, despite low spatial resolution, of about $1-2 \mu \mathrm{m}$, indicate that swift ions many damage the near-surface region. To confirm this, a slow positron beam technique was used to study the irradiated samples. The results are shown in Fig. 4. Note that the mean depth of implanted positrons is marked on the upper axis, this depth was calculated using the following relationship, $\bar{z}(\mathrm{~nm})=8.61 \times E^{1.372}$, where $E$ is the input positron energy in $\mathrm{keV}$, shown in bottom axis [31]. The total range of this measurement is from $10 \mathrm{~nm}$ to $1.3 \mu \mathrm{m}$. The open diamonds represent the dependency of the $S$-parameter versus the positron incident energy obtained for the reference, well-annealed, non-irradiated sample. It is well-known that the S-parameter reflects the annihilation 
mean implantation positron depth $(\mathrm{nm})$

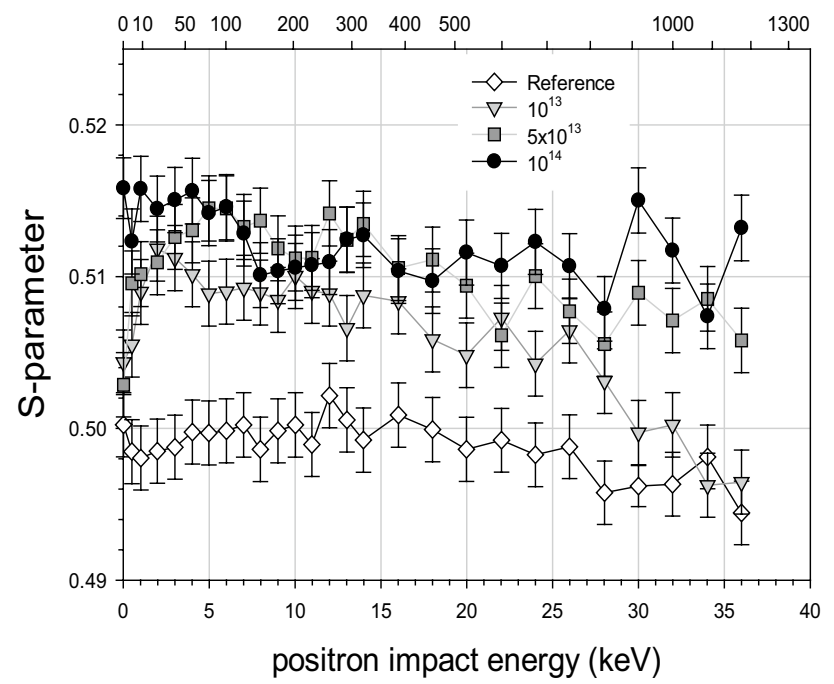

Fig. 4 The measured annihilation line shape, S-parameter, as a function of the positron incident energy for Bi samples irradiated with $\mathrm{Xe}^{26+}$ ions with three different doses. The top axis represents the mean positron implantation depth

of positrons with low momentum electrons. The latter is present in open volume defects, such as vacancy and its clusters. Therefore, this parameter similar to the mean positron lifetime is sensitive to these defects in measured samples. In Fig. 4, the value of the S-parameter for the virgin sample is lower than for the samples subjected to irradiation. There is also a tendency that the S-parameter increases in value as the dose increases. It is not clearly visible in Fig. 1b, where the values of the mean lifetime of positron on the entrance surface for all these samples are almost equal. We can conclude that despite the high energy of implanted $\mathrm{Xe}^{26+}$ ions and consequently, their deep penetration, the surface close to the surface is strongly damaged. What's more, the level of this damage depends on the dose.

Values of the measured S-parameter that are presented in Fig. 4 do not change significantly with the increase of positron energy, the dependencies are almost flat. It results from the fact that the value of parameter $S$ on the surface does not differ significantly from the value in the interior. It makes it impossible to obtain quantitative information, e.g., values of the positron diffusion length. Thus, let us remain on the qualitative analysis of the results from Fig. 4.

\section{Final remarks}

Taking into account the results in this article, we can summarize our current research on LRE in the following way.

ELD accompanies IL in $\mathrm{Bi}$, its occurrence is the result of ion presented implantation, however, that ELD was not observed for $\mathrm{Cu}$ and $\mathrm{Ti}$ irradiated with $\mathrm{Xe}^{26+}$ ions $[11,14]$. This may indicate that the properties of the target material may also affect ELD formation. The dose seems to be an important factor responsible for the distribution of defects in the ELD, and their depth distribution.

Processes occurring in IL are the source of defects in ELD. Dynamic or static stress occurring during implantation is very likely, the value of which exceeds the yield strength. This stress must cause additional IL defects that overlap defects resulting from ionization and nuclear collision with nuclei of atoms of the target material. This can explain why in all investigated cases the depth distribution of defects does not correspond to the vacancy depth distribution obtained from the SRIM simulation. The defect concentration seen by positrons is higher near the entrance surface and then gradually decreases according to the reconstruction that at least remains constant throughout the depth. There is no maximum observed at the depth at which the Bragg peak should be located. This was observed in all metals tested [10-14] and also this time for semimetal in Bi.

The question arises whether processes at the atomic scale or in global processes induce stress, such as an increase in temperature when the current with ions hits the target. Thermal lattice expansion can be responsible for tensile stress. Nevertheless, an increase in temperature enhances the migration of defects, their coalescence, rearrangement or annealing and finally reduces stress. It can be taken into account that stress can also be accompanied by ionization, causing the local melting of the material according to the thermal spike model. It locally expands the crystalline lattice and plastically deforms the region also outside the spike. It can be dynamic stress, which works only in a very short time of a few ns or less, but it is enough to leave a lot of defects.

Ion implantation is used in doping technology for the production of flat devices mainly for electronics [32]. The fact that this implantation leads to radiation damage, i.e., defects' generation, can counteract the desired effect of doping. In addition, according to this study, defects may also be generated outside IL, this may affect the substrate or other structures below. In particular, it can affect the electrical resistivity that is sensitive to the presence of defects at the atomic scale and can be modified due to this effect. The use of a high dose may enhance this effect. This can be important when the implantation takes place in a multilayer system, because implanting ions into the top layer can generate defects and modify properties in other layers below if they exist.

\section{Conclusions}

The measurement of the depth distribution of the positron lifetime in pure Bi irradiated with swift $\mathrm{Xe}^{26+}$ ions of $167 \mathrm{MeV}$ revealed that ions induce open volume defects not 
only in the implantation layer, but also beyond this layer at depths exceeding the projectile range. For the highest dose it is about $28 \mu \mathrm{m}$, whereas the projectile range obtained from the SRIM/TRIM simulations is only about $13.2 \mu \mathrm{m}$. The total thickness of the damage layer increases with dose increase. In damaged layers, clusters which consist of eight or more vacancies are generated defects, according to ab initio calculations of a positron lifetime in vacancy clusters performed for $\mathrm{Bi}$. Also near the entrance surface region with defects induced by implantation is clearly visible. It suggests that not only inelastic nuclear collision cascades, but ionization and excitation of electrons also induce defect generation. We suggested that the occurrence of the tensile stress, results from, for instance, thermal expansion and/or swelling may be responsible for the long range of defect distribution.

Due to low range of induced defects in comparison to the positron implantation range, two approaches for reconstructing the real mean positron depth of life were proposed. In the first approach, the reconstruction was done using only the measured values. Despite the low accuracy, it can be helpful in detecting the actual defect distribution. In the second approach, the proposed depth profile after convolution with positron implantation profile was fitted to the experimental data. A step-like function, as a proposed profile, is able to describe the experiment results in a satisfactory manner.

Acknowledgements The authors express their gratitude to V.A. Skuratov for his technical help and assistance in ion irradiation.

Open Access This article is distributed under the terms of the Creative Commons Attribution 4.0 International License (http://creativeco mmons.org/licenses/by/4.0/), which permits unrestricted use, distribution, and reproduction in any medium, provided you give appropriate credit to the original author(s) and the source, provide a link to the Creative Commons license, and indicate if changes were made.

\section{Appendix 1}

After emission from the nucleus, the positron has enough energy to penetrate a certain region before it slows down to thermal energy and finally annihilates. The depth distribution of thermalized positrons called the implantation profile: $p(x)$, where $x$ is the depth from the positron source, causes that the measured value of the positron mean lifetime, which characterizes the local properties of the sample, is averaged over this profile. For the case when the sample is etched and the layer of thickness $z$ is removed, the following integral equation links both: the actual: $\bar{\tau}(x)$ and the measured $\bar{\tau}_{m}(z)$ value of the mean positron lifetime as follows:

$\bar{\tau}_{\mathrm{m}}(z)=\int_{z}^{\infty} p(x-z) \bar{\tau}(x) \mathrm{d} x$.

When that implantation profile is expressed as the exponential decay function with the linear absorption coefficient $\mu$ :

$p(x)=\mu \exp (-\mu x)$

the solution of Eq. (4) is as follows:

$\bar{\tau}(z)=\bar{\tau}_{\mathrm{m}}(z)-\frac{1}{\mu} \frac{\mathrm{d} \bar{\tau}_{\mathrm{m}}(z)}{\mathrm{d} z}$.

Thus, the measured mean positron lifetime reconstitutes its actual value minus the gradient correction term. Examples of typical relationships of $\bar{\tau}(z)$ and according to Eq. (4) corresponding them $\bar{\tau}_{\mathrm{m}}(z)$ is presented in Fig. 5. The examples were chosen for a possible depth distribution of defects in the implanted layer and thus possible $\bar{\tau}(z)$ dependencies. In all cases, except Fig. $5 \mathrm{~d}, \bar{\tau}_{\mathrm{m}}(z)$ (solid lines) decreases with increasing depth to the thickness of
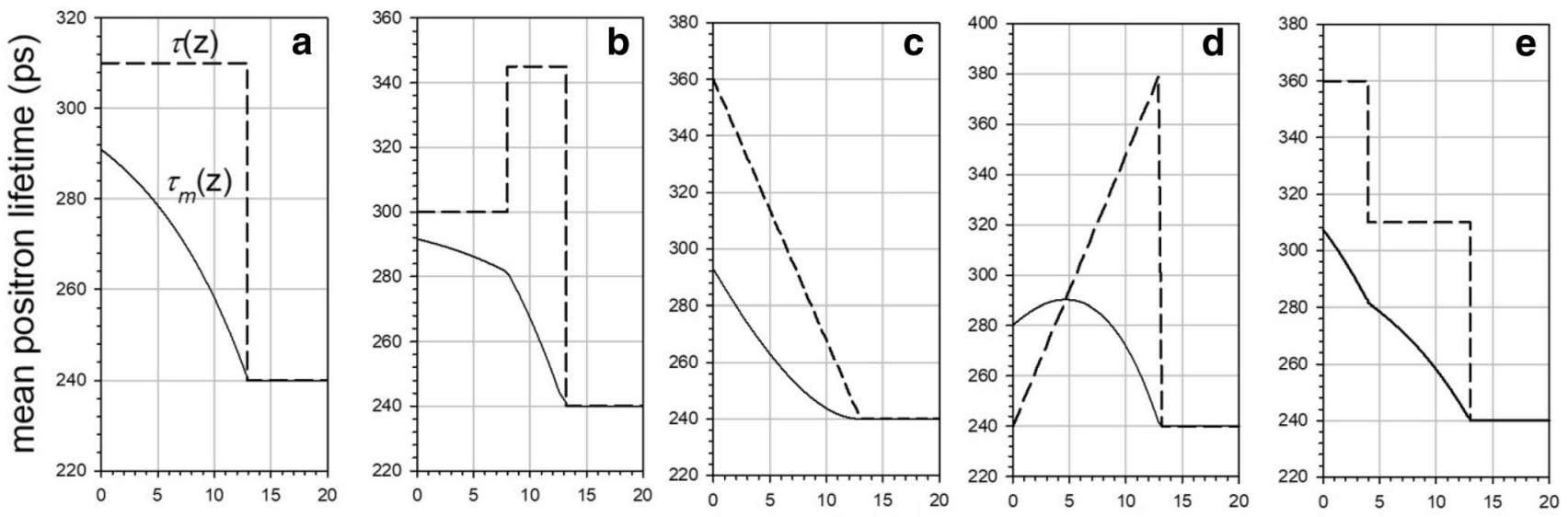

\section{depth-z $(\mu \mathrm{m})$}

Fig. 5 The examples of typical expected local depth dependencies of: $\bar{\tau}(x)$ (dashed line). In $\mathbf{a}$ it is a step function, $\mathbf{b}$, e two steps function, $\mathbf{c}$ linear descending function, $\mathbf{d}$ linear increasing function and according Eq. (4) corresponding them $\tau_{\mathrm{m}}(z)$ dependencies (solid line), assuming $\mu=1000 \mathrm{~cm}^{-1}$ 
the implanted layer, here $13 \mu \mathrm{m}$, but the decreases reflect the type of dependence assumed.

In an experiment, only limited number of values of $\bar{\tau}_{\mathrm{m}}(z)$ are available at different depths, so Eq. (6) is difficult to apply to obtain the actual mean positron value at certain depths. However, it may be useful in its approximation form, as follows:

$\langle\bar{\tau}(z: z+\Delta z)\rangle \simeq \bar{\tau}_{\mathrm{m}}(z+\Delta z)-\frac{\bar{\tau}_{\mathrm{m}}(z+\Delta z)-\bar{\tau}_{\mathrm{m}}(z)}{w}$.

Thus having values of $\bar{\tau}_{\mathrm{m}}$ measured at the depth $z$ and $z=z+\Delta z$, one can determine the average values of the mean positron lifetime in the layer at the depth between $z$ and $z+\Delta z$. For $\Delta z \rightarrow 0$ this value tends to the local mean positron lifetime at a depth $z$, Eq. (6). In this equation $w$ represents the fraction of positrons which annihilate in the layer at the depth between $z=0$ and $z=\Delta z$, note that for small value of $\Delta z$, and taking into account Eq. (5), it can be is expressed as follows: $w \approx \mu \Delta z . \Delta z$ can be equal to the thickness of the etched layer of about $2 \mu \mathrm{m}$ in our experiment, however it can be too small to obtain $\langle\bar{\tau}(z: z+\Delta z)\rangle$ with satisfactory accuracy. Note that the accuracy of this value depends on the inverse of $w$ value, which is much less than unity, in addition, this value is very sensitive to the depth measurement accuracy. Considering a larger thickness, for example $5 \mu \mathrm{m}$ or more, it is good to determine the positron fraction in such a layer using, for instance the LYS-1 code [25, 26]. This code allows the determination of the fraction of positrons emitted from ${ }^{22} \mathrm{Na}$ and ${ }^{68} \mathrm{Gel}^{68} \mathrm{Ga}$ in a stack of layers with different thicknesses.

Reconstruction can also be performed using the following equation:

$\langle\bar{\tau}(z: z+\Delta z)\rangle \simeq \frac{\bar{\tau}_{\mathrm{m}}(z)}{w}+\sum_{i=1} \mathrm{e}^{-i w}\langle\bar{\tau}(z+i \Delta z: z+(i+1) \Delta z)\rangle$.

In this case, we must use the measured value of the mean positron lifetime at depth $z$, i.e., $\bar{\tau}_{\mathrm{m}}(z)$, and local values in the deeper layers: $\langle\bar{\tau}(z+(i-1) \Delta z: z+i \Delta z)\rangle$, $i=1,2 \ldots$, These values can be determined earlier, starting reconstruction from the deepest layer and following in the direction of the entrance surface. However, the uncertainty will be reproduced at every stage.

\section{Appendix 2}

There is a relationship between the number of vacancies in the cluster and the lifetime of positrons trapped there. That is in metals and other materials. This relationship has been determined using ab initio theoretical calculations

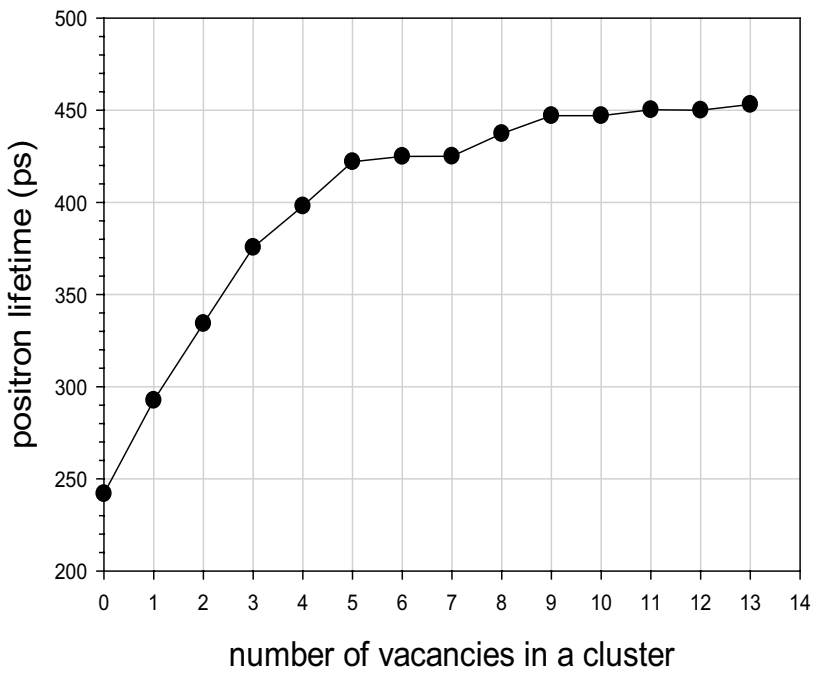

Fig. 6 Positron lifetime as a function of the number of vacancies in a cluster in Bi calculated using ABINIT v 8.4.4 code [34]

but is accepted by the experimentalists. For Bi numerical calculations were carried out using the PAW formalism as implemented in ABINIT v 8.4.4 for the purpose of this paper $[33,34]$. PAW potential for Bi was chosen from the available in the pseudopotential repository $[35,36]$. We used Perdew-Burke-Ernzerhof (PBE) potentials generated using the Vanderbilt pseudopotential generation code. K-point space was sampled by $2 \times 2 \times 2$ Monkhorst-Pack (MP) set, which corresponds to $4 \mathrm{k}$-points [37]. The positron lifetime computations were performed on 54 atoms Bi supercell. A specific supercell was constructed to introduce Bi vacancy around the central atom. For the calculations, the enhancement factor from Boroński-Nieminen in the RPA limit parametrization was used [38]. In Fig. 6 the positron lifetime as a function of the number of vacancies in a cluster is depicted. Similar dependency was obtained by other authors for other metals.

\section{References}

1. G.S. Was, Fundamentals of Radiation Damage Using Ion Beam (Springer, New York, 2007)

2. S. Mammeri, S. Ouichaoui, H. Ammi, C.A. Pineda-Vargas, A. Dib, M. Msimanga, Nucl. Instrum. Methods Phys. Res. B 354, 235 (2015)

3. Ch Dufour, F. Beuneu, E. Paumier, M. Toulemonde, Europhys. Lett. 45, 585 (1999)

4. J.F. Ziegler, The Stopping and Range of Ions in Solids (Pergamon, New York, 1985)

5. C. Lu et al., Sci. Rep. 6, 19994 (2016)

6. Yu.P. Sharkeev, N.V. Girsova, A.I. Ryabchikov, E.V. Kozlov, O.B. Perevalova, I.G. Brown, X.Y. Yao, Nucl. Instrum. Methods Phys. Res. B 106, 532 (1995) 
7. R. Krause-Rehberg, H.S. Leipner, Positron Annihilation in Semiconductors (Springer, Berlin, 1999)

8. L. Liszkay, P.M. Gordo, K. Havancsák, V.A. Skuratov, A. de Lima, Zs. Kajcsos, Mater. Sci. Forum 445-446, 138 (2004)

9. P.G. Coleman (ed.), Positron Beams and Their Applications (World Scientific, Singapore, 2000)

10. P. Horodek, J. Dryzek, V.A. Skuratov, Rad. Phys. Chem. 122, 60 (2016)

11. P. Horodek, J. Dryzek, V.A. Skuratov, Vacuum 138, 15 (2017)

12. J. Dryzek, P. Horodek, V.A. Skuratov, Acta Phys. Polon. A 132, 1585 (2017)

13. J. Dryzek, P. Horodek, M. Dryzek, Appl. Phys. A 124, 451 (2018)

14. P. Horodek, J. Dryzek, Surf. Coat. Technol. 355, 247 (2018)

15. Yu.P. Sharkeev, E.V. Kozlov, A.N. Didenko, S.N. Kolupaeva, N.A. Vihor, Surf. Coat. Technol. 83, 15 (1996)

16. Yu.P. Sharkeev, E.V. Kozlova, Surf. Coat. Technol. 158- 159, 219 (2002)

17. A. Polanski, S. Petrochenkov, W. Pohorecki, Nucl. Instrum. Methods Phys. Res. A 562, 764 (2006)

18. S. Mammeri, M. Msimanga, A. Dib, H. Ammi, C.A. Pineda-Vargas, Surf. Interface Anal. 50, 328 (2018)

19. S. Mammeri, S. Ouichaoui, H. Ammi, H. Hammoudi, C.A. Pineda-Vargas, Nucl. Instrum. Methods Phys. Res. B 269, 909 (2011)

20. M.A. Monge, J. del Rio, J. Phys. Condens. Matter 6, 2643 (1994)

21. N. Djourelov, M. Misheva, J. Phys. Condens. Matter 8, 2081 (1996)
22. J.M.Campillo Robles, E. Ogando, F.J. Plazaola, J. Phys. Condens. Matter 19, 176222 (2007)

23. J. Dryzek, D. Singleton, Nucl. Instrum. Methods Phys. Res. B 252, 197 (2006)

24. L.Yu. Dubov, Yu.A. Akmalova, S.V. Stepanov, Yu.V. Funtikov, Tu.V. Shtotsky, Acta Phys. Polon. A. 132, 1482 (2017)

25. J. Dryzek, K. Siemek, J. Appl. Phys. 114, 074904 (2013)

26. See: http://www.ifj.edu.pl/mdryzek/index.html. Accessed 1 Aug 2013

27. R.A. Kinomura, T. Suzuki, N. Ohdaira, K. Oshima, Y. Ito, T. Kobayashi, Iwai, J. Phys. Conf. Ser. 262, 012029 (2011)

28. J. Dryzek, Tribol. Lett. 40, 175 (2010)

29. M. Domkus, L. Pranyavichus, Mechanical stresses in implanted solids (Mokslas, Vilnus, 1990), p. 158

30. E.P. EerNisse, S.T. Picraux, J. Appl. Phys. 48, 9 (1977)

31. J. Dryzek, P. Horodek, Nucl. Instrum. Methods Phys. Res. B 266, 4000 (2008)

32. R.W. Hamm, M.E. Hamm, Industrial Accelerators and Their Applications (World Scientific, Singapore, 2012)

33. P.E. Blöchl, Phys. Rev. B 50, 17953 (1994)

34. ABINIT 8.4.4 code. http://www.abinit.org. Accessed 2 June 2017

35. https://www.physics.rutgers.edu/gbrv/. Accessed 23 Sept 2015

36. K.F. Garrity, J.W. Bennett, K.M. Rabe, D. Vanderbilt, Comput. Mater. Sci. 81, 446 (2014)

37. D. Vanderbilt, Phys. Rev. B 41, 7892 (1990)

38. E. Boroński, R. Nieminen, Phys. Rev. B 34, 3820 (1986) 